\title{
Bilinguisme ou plurilinguisme
}

Les droits de l'homme et les langues étrangères

Bilingualism or multilingualism. Human rights and the teaching of foreign

languages

Bilingüismo o plurilingüismo. Los derechos humanos y los idiomas extranjeros

\section{Cecilia Braslavsky}

\section{QpenEdition}

\section{Journals}

Édition électronique

URL : http://journals.openedition.org/ries/2611

DOI : 10.4000/ries.2611

ISSN : 2261-4265

Éditeur

Centre international d'études pédagogiques

Édition imprimée

Date de publication : 1 décembre 1999

Pagination : 59-65

ISSN : 1254-4590

\section{Référence électronique}

Cecilia Braslavsky, "Bilinguisme ou plurilinguisme », Revue internationale d'éducation de Sèvres [En ligne], 24 | 1999, mis en ligne le 23 novembre 2012, consulté le 10 décembre 2020. URL : http:// journals.openedition.org/ries/2611; DOI : https://doi.org/10.4000/ries.2611

(c) Tous droits réservés 


\title{
Bilinguisme ou plurilinguisme
}

\author{
Les droits de l'homme et les langues étrangères \\ Cecilia Braslavsky \\ Professeur en sciences de l'éducation à l'université nationale \\ de Buenos Aires, consultant auprès de l'UNESCO
}

\section{Le plurilinguisme comme une opportunité pour faire réaliser le droit de vivre ensemble}

Un des premiers droits des hommes et des femmes est de vivre ensemble. Et pour vivre ensemble, la paix est une priorité. Nous vivons dans un monde de guerre. Mais le pire, c'est que nous n'en avons même pas conscience. Presque tous les jours, les journaux font état d'une violation de frontières, de crimes commis par une guérilla, de l'oppression ou du soulèvement d'une minorité. Beaucoup de guerres sont le résultat de la survivance de conflits et de tensions demeurés latents durant des décennies. D'autres s'expliquent par les particularités du contexte international actuel. La première catégorie de guerre est à mettre en relation avec l'émergence du communautarisme. Dans la seconde catégorie, certaines guerres sont la conséquence du renforcement d'un monde soidisant unifié, "globalisé ». En tout cas, en toile de fond de toutes les guerres se trouve l'absence de reconnaissance de l'autre comme un sujet qui a le droit de vivre et de se développer, d'un autre avec lequel on peut vivre ensemble. De plus en plus, nous sommes face à un monde perçu à la fois comme unitaire et fragmenté, désagrégé, fait de groupes humains aux identités multiples qui s'épanouissent parallèlement et aspirent à la " tolérance " plutôt qu'à la reconnaissance de l'autre et donc à l'enrichissement mutuel.

On assiste à une refonte de l'État. Beaucoup s'affaiblissent selon des processus très variés. Parmi ceux-là quelques-uns se divisent, d'autres se réunifient. D'autres commencent à envisager des formes inédites de réorganisation territoriale, comme la Colombie qui transforme les groupes guérilleros en coadministrateurs de la chose publique. D'autres encore choisissent des alternatives moins déchirantes, mais dont l'impact est tout aussi fort. L'Espagne assume avec ferveur le rôle politique des provinces autonomes ainsi que le droit de ses peuples à enseigner et étudier dans leur propre langue alors que pendant des années celles-ci ont été oubliées et se sont appauvries : le basque, le catalan ou le galicien. Dans la plupart de ces cas, le processus de construction de l'identité 
individuelle fait intervenir la référence à la communauté. Parfois, dans ce processus, la communauté semble même être le référent unique, privilégié, voire exclusif, de construction de cette identité.

Si nous envisagions cette tendance à l'extrême et, dans un grand nombre de pays, nous pourrions aller jusqu'à hasarder que, pour préserver l'unité, on peut en arriver à ne garder que l'anglais. Non pas l'anglais comme moyen d'accéder aux traditions politiques ou aux manifestations culturelles des peuples qui l'ont construit, ni comme moyen de renforcer la capacité de compréhension de leur propre syntaxe communicative par comparaison avec d'autres, mais l'anglais de la raison instrumentale dissocié de tout processus de subjectivisation. L'anglais du marché international, de l'expression de quelques formes dynamiques de production de connaissance dans quelques domaines clés.

La défense du droit à l'identité communautaire, d'une part, et la présence de l'économie et de la technologie globales comme forces dominantes avec leurs lois et leurs règles propres, d'autre part, agissent dans certains pays comme une tenaille qui pousse vers la configuration d'un modèle de promotion d'un bilinguisme orienté vers la reconnaissance et l'approfondissement de l'apprentissage à la fois d'une langue communautaire et d'une langue instrumentale ; parfois en conquérant une partie de l'espace curriculaire auparavant fortement consacré à l'enseignement de la seule langue ou d'une deuxième langue nationale.

Les deux bras de la tenaille ne sont pas eux-mêmes contestables. Après des années d'acculturation des peuples indigènes d'Amérique latine et de progrès certains qui démontrent que l'accès aux formes supérieures de la pensée et à la langue nationale est facilité s'il passe par la langue maternelle, il serait réducteur de postuler l'abandon des efforts des autorités publiques qui prétendent octroyer aux langues vernaculaires une place dans le système scolaire. Et face à l'existence incontestable d'une économie et d'une technologie globalisées, il serait en même temps suicidaire de suggérer que l'on puisse refuser à des enfants et des jeunes du Xxe siècle la possibilité d'apprendre l'anglais pour la communication internationale.

Par conséquent, la question qu'il convient de se poser est de savoir si une politique orientée volontairement ou par défaut vers la promotion du bilinguisme, tel que je l'ai exposé ci-dessus, est ce qui convient de mieux pour réaliser les droits des femmes et des hommes. Ma réponse est non. Parce que le bilinguisme vers lequel s'orientent plus ou moins consciemment les politiques éducatives de nombreux pays renforce une double dépendance de la tradition communautaire, du marché et des techniques qui autorise seulement l'épanouissement des producteurs et des consommateurs et non pas le développement complet des citoyens.

Cette double dépendance empêche, ou tout du moins limite, la possibilité de transformer ce monde de guerres et d'inégalités en un monde de paix. Avec le bilinguisme des langues communautaires et de l'anglais du marché et de la 
technique, les seules choses communicables seraient les règles de production et de circulation des biens du capital et de l'information. Mais pas la multiplicité des expériences vécues dans la diversité des cultures existantes. Pas les sentiments et les émotions des autres communautés. Pas les pratiques d'organisation collective envisagées depuis une multitude de perspectives. Pas les formes et les limites des réalisations des droits des hommes et des femmes dans des conditions différentes.

C'est pour cela qu'il paraît nécessaire de plaider en faveur de la permanence et de l'approfondissement des politiques publiques qui œuvrent pour la promotion du multilinguisme, c'est-à-dire pour la promotion de la présence d'une multiplicité de langues dans le cadre des contenus de l'éducation. Il s'agit donc de promouvoir le multilinguisme comme affirmation humaniste. Dans ce multilinguisme, il s'agit aussi de concevoir l'enseignement des langues nationales, non pas contre l'enseignement des langues communautaires ou de l'anglais, mais comme une stratégie qui permette aux individus de disposer de davantage de moyens pour créer des passerelles entre les communautés et la culture mondiale et pour livrer les batailles internes contre le mépris des autres, qu'ils soient étrangers ou simplement différents au sein d'un même pays.

\section{Le plurilinguisme et les inégalités entre les pays}

Mais nous vivons aussi dans un monde où les inégalités augmentent et, dans ces conditions, les langues nationales autres que l'anglais tendent dans la majorité des pays, en particulier en Amérique latine, à être confinées dans le rôle de reproduction des avantages comparatifs préexistants ou de la consommation somptuaire en apparence.

En Europe, il y a des pays où les langues étrangères nationales occupent dans l'enseignement secondaire entre 17 et $26 \%$ du temps scolaire (Pays-Bas, Allemagne). Dans quelques uns, les contenus des langues étrangères nationales prennent plus de temps que les contenus de mathématiques et de sciences. Mais en Amérique latine, seuls 8 à $9 \%$ du temps scolaire sont consacrés à l'enseignement des langues étrangères. La différence est beaucoup plus importante qu'il n'y paraît parce que le temps total disponible est très différent. A la fin de neuf années de scolarisation, les jeunes d'Argentine ou du Mexique ont eu 6580 ou 6600 heures de classe ; en Allemagne 7080 heures; en France 8400 et aux Pays-Bas 9700.

Le manque de temps scolaire, la pression des communautés et les difficultés à créer des alternatives par l'utilisation des nouvelles technologies conduisent les pays vers le bilinguisme pauvre. En Argentine, par exemple la population est de 35 millions d'habitants. Les communautés indiennes comptent près de 300000 personnes. Ces dernières ont demandé et obtenu, pour la première fois, la possibilité d'apprendre leurs langues à l'école : le wichi, le toba, l'araucant et le mapuche. 
Parallèlement la plus importante province du pays a décidé, pour l'enseignement des langues étrangères, de n'autoriser et de ne financer dans les écoles publiques que l'anglais. Les autorités ont décidé de "recycler » les professeurs de français et d'italien et de leur faire enseigner l'espagnol. Un long processus de consensus a été nécessaire entre les ministères de l'Éducation des vingtquatre provinces du pays pour éviter la généralisation de cette politique dans tous le pays. Les négociations ont conduit au postulat suivant : tout le monde doit avoir la chance d'apprendre à l'école trois niveaux de 240 heures d'une langue autre que la langue nationale. L'un de ces niveaux doit être l'anglais pour la communication internationale; les autres peuvent être les langues indigènes, ou bien un approfondissement de l'anglais, ou bien encore d'autres langues étrangères dont le choix ne se limiterait pas au français ou à l'italien comme dans le passé.

La politique menée par la province en question était liée aux élections : selon les sondages, les électeurs attendaient de l'école qu'elle se concentre sur un enseignement " anglais et informatique ". Pas de sciences, pas de plurilinguisme, pas de sports, pas d'arts. Pourquoi ? Les raisons sont nombreuses, je n'en reprendrai qu'une : le problème de modèle pédagogique d'enseignement des langues étrangères dans les écoles secondaires.

\section{Critères pédagogiques et didactiques pour la fertilité formative de l'enseignement des langues étrangères}

Nous visitons souvent des écoles. Au cours de ces visites, nous avons souvent pu assister à des cours de langue étrangère. Chaque fois, nous sommes témoins de stéréotypes classiques. Les Français sont sensibles, fins et élégants. Les Allemands sont rigoureux, méthodiques et ordonnés. Les Anglais sont courtois et ponctuels. Les Italiens sont impulsifs et font de bons amants.

Les livres à partir desquels les Argentins ont appris le français au cours de leur scolarisation dans les établissements destinés à la formation des élites montraient le plan de Paris, la vie quotidienne d'une famille parisienne et comment aller de l'Arc de Triomphe à la Tour Eiffel. Les adultes qui parlent français ont lu Molière, Balzac et d'autres classiques de la littérature française avant leurs dix-sept ans. Mais ils n'ont jamais rien appris sur les invasions napoléoniennes, le rôle de la France en Afrique - et encore moins en Amérique -, l'existence du Québec et sa situation particulière dans la Confédération canadienne, la trajectoire du nationalisme français et ses relations avec la politique latino-américaine dans les années vingt, la question algérienne contemporaine et les tensions sociales de l'après-guerre. Le français que l'on enseignait et enseigne encore dans les collèges transpirait le stéréotype, la naphtaline, le mensonge et le secret. 
Quand les élites voyagent en France, elles découvrent les différences entre les stéréotypes et la réalité : il y a des Français cultivés - la minorité - et d'autres qui n’ont jamais lu ni Molière ni Balzac; la ville est à la fois dorée et grise ; dans le métro, il y a beaucoup d'Arabes et d'Africains. Et, après tant d'années d'études, ils ne savent même pas pourquoi.

La même chose, si ce n'est pire, s'est produite avec l'anglais. Ce n'est ni Balzac ni Molière, mais Shakespeare. Par contre, aujourd'hui dans la plupart des livres et des cours pour les couches populaires, on enseigne un vocabulaire immédiatement utilisable : tableau, gomme, maître, élève. Il est difficile d'évaluer si c'est mieux.

En tout cas, l'expérience des élites n'est pas la pire. Elles baragouinent dans les deux langues des phrases curieuses où se mêlent des expressions empruntées au vocabulaire du XVII ${ }^{e}$ siècle mais aussi du XXe siècle sur des thèmes propres à la grande culture, inappropriés, pour mettre en évidence ce qui est bien en France, en Grande-Bretagne ou aux Etats-Unis. Elles n'ont pas aujourd'hui perdu leur temps. Mais j'ai pu constater, alors que j'étais à la recherche de solutions alternatives, que de nombreux jeunes issus d'établissements publics d'enseignement ne savent, au terme d'au moins 240 heures d'apprentissage d'une langue étrangère, au cours des trois ou quatre ans de collège, ni répondre au téléphone, ni dire au revoir.

Il y a sûrement beaucoup d'explications à donner qui mettent en évidence les mauvaises conditions d'enseignement dans un certain nombre d'établissements et, surtout, dans les établissements publics destinés aux couches populaires des pays latino-américains. De mauvaises conditions d'enseignement sont à l'origine des problèmes détectés ainsi que d'autres problèmes encore plus graves. On peut parler d'un modèle d'établissement éducatif balkanisé et rigide dans lequel il y a peu, ou pas du tout, de matériel pédagogique à la disposition des enseignants, des salaires de misère et une formation médiocre du corps enseignant. Cependant il est possible qu'il y ait aussi des choses à revoir dans la sélection des contenus et dans la manière d'enseigner.

J'essaierai de présenter quatre critères qui permettent de faire de l'enseignement des langues étrangères à l'école l'instrument d'une politique éducative pour la promotion du plurilinguisme, comme une stratégie pour la citoyenneté et non comme une représentation théâtrale du temps perdu ou l'acquisition d'un vernis pour montrer qu'on est « de la haute », qu'on a de la « distinction ».

D'abord, à travers l'apprentissage d'une langue étrangère, les enfants et les jeunes peuvent découvrir que dans le monde il y a d'autres personnes qui parlent, sentent, pensent et agissent différemment. Il s'agit de faire découvrir aux enfants et aux jeunes que ces " autres " ne sont pas des stéréotypes de personnes tout à fait bonnes ou tout à fait mauvaises, et qu'ils ne font pas partie d'un ensemble homogène, charmant et épargné de tout confit. Ces «autres " sont des être humains en constante interaction, avec leurs productions sociales, artifi- 
cielles et symboliques. Ils font partie d'une culture avec ses grandeurs et ses décadences. Et cette culture change de sens selon la lorgnette à travers laquelle on la regarde ou l'endroit à partir duquel on l'observe. Mais, par ailleurs, les enfants et les jeunes apprennent une langue en tant qu'enfants ou jeunes. Ils ne l'apprennent pas comme adultes. Ils font partie d'une communauté et de ce marché globalisé.

C'est pour cette raison que le second critère est que les enfants et les jeunes puissent retrouver dans les autres des éléments de leur moi, de leur moi tel qu'il est vraiment. S'interroge-t-on, quand on enseigne les langues étrangères, sur le sens que revêt ce que l'on propose de lire ou de raconter pour ceux que l'on place dans cette situation ? Proposons-nous des contenus sur l'éclatement des familles, la drogue, l'oisiveté et la construction de l'identité sexuelle des jeunes dont nous étudions la langue ? Parlons-nous des conflits résultant des inégalités, de la crise de la représentativité que traversent la politique et les institutions, de la violence de l'état, de la guerre, des nouvelles manifestations religieuses ? Autrement dit, proposons-nous des contenus qui aident l'enfant ou le jeune pendant son apprentissage de la langue étrangère à rencontrer l'autre ?

Enfin, facilitons-nous la construction d'un «nous » multilingue et pardessus tout multiculturel et divers à la fois ? A certains niveaux on sait que tout ce qui se passe actuellement dans le monde a quelque chose à voir avec autre chose qui a lieu à des milliers de kilomètres de là. Ainsi le troisième critère est que l'enseignement des langues étrangères fait partie d'une quête d'un « nous » qui dépasse le champ de la communauté de résidence et de référence et qui permet de repersonnaliser le contexte global unilatéralement représenté et vécu comme un marché. Il y a des " autres " égaux mais différents. Proposons-nous comme des contenus les techniques communautaires pour faire des recherches comparatives: " vivre à côté de et tolérer ", "lutter contre " ou/et " vivre à côté de et tolérer " ?

Ce que j'ai exposé jusqu'à présent est étroitement lié au choix des thèmes et des problèmes de conversation. Parce qu'à mon avis l'enseignement d'une langue étrangère devrait être avant tout ceci : une grande opportunité de dialogue. Et ceci correspond au quatrième critère : l'enseignement d'un langue étrangère entendu comme une grande opportunité de dialogue passe par la capacité de combiner la mémoire, la raison, le plaisir et l'émotion, afin qu'il y ait en même temps une formation rigoureuse et un espace réservé à la recréation à travers la création des éléments de la propre culture communautaire et nationale et des cultures des langues apprises.

C'est seulement de cette manière que l'enseignement des langues étrangères sera plus efficace et atteindra ces objectifs qui valent vraiment la peine : rencontrer l'autre, nous retrouver dans l'autre et nous rencontrer tous dans un 
monde qui de cette manière aura plus de chance - mais hélas point la garantie d'être plus pacifique et, en même temps, plus équitable et plus divers. Si l'on peut montrer et démontrer cela, peut être aura-t-on la chance de maintenir et d'approfondir les contenus de plusieurs langues nationales dans les écoles des différents pays pour développer la compétence d'être plurilingue. Parce qu'être plurilingue est l'une des conditions au développement du droit à une vraie citoyenneté, c'est-à-dire à une citoyenneté à quatre niveaux : communauté, nation, échanges entres les nations, dans le monde globalisé. 